This is the peer reviewed version of the following article: Lear, R., Riga, C., Godfrey, A. D., Falaschetti, E., Cheshire, N. J., Van Herzeele, I., Norton, C., Vincent, C., Darzi, A. W. and Bicknell, C. D. (2016) Multicentre observational study of surgical system failures in aortic procedures and their effect on patient outcomes. British Journal of Surgery, 103(11), pp. 1467-1475., which has been published in final form at https://doi.org/10.1002/bjs.10275 . This article may be used for non-commercial purposes in accordance with Wiley Terms and Conditions for Use of Self-Archived Versions. 


\section{Multicentre observational study of surgical system failures in aortic procedures and their effect on patient outcomes}

R. Lear ${ }^{1,4}$, C. Riga ${ }^{1,4}$, A. D. Godfrey ${ }^{1}$, E. Falaschetti ${ }^{2}$, N. J. Cheshire ${ }^{1}$, I. Van Herzeele ${ }^{7}$, C. Norton ${ }^{4,5}$, C. Vincent ${ }^{6}$, A. W. Darzi ${ }^{1,3}$ and C. D. Bicknell ${ }^{1,3,4}$, on behalf of the LEAP study collaborators*

${ }^{1}$ Department of Surgery and Cancer, ${ }^{2}$ Clinical Trials Unit and ${ }^{3}$ Centre for Health Policy, Imperial College London, ${ }^{4}$ Imperial College Healthcare NHS Trust, and ${ }^{5}$ Faculty of Nursing and Midwifery, King's College London, London, and ${ }^{6}$ Department of Experimental Psychology, Medical Sciences Division, Oxford University, Oxford, UK, and ${ }^{7}$ Department of Thoracic and Vascular Surgery, Ghent University Hospital, Ghent, Belgium

Correspondence to: Ms R. Lear, Division of Surgery, Department of Surgery and Cancer, Imperial College London, St Mary's Campus, London W2 1NY, UK (e-mail:

r.lear12@imperial.ac.uk)

Background: Vascular surgical care has changed dramatically in recent years with little knowledge of the impact of system failures on patient safety. The primary aim of this multicentre, observational study was to define the landscape of surgical system failures, errors and inefficiency (collectively termed failures) in aortic surgery. Secondary aims were to investigate determinants of these failures and their relationship with patient outcomes.

Methods: Twenty vascular teams at ten English hospitals trained in structured, self-reporting of intraoperative failures (phase I). Failures occurring in open and endovascular aortic procedures were reported in phase II. Failure details (category, delay, consequence), demographic information (patient, procedure, team experience) and outcomes were reported. 
Results: There were strong correlations between the trainer and teams for the number and type of failures recorded during 88 procedures in phase I. In 185 aortic procedures, teams reported a median of 3 (i.q.r. 2-6) failures per procedure. Most frequent failures related to equipment (unavailability, failure, configuration, desterilization). Most major failures related to communication. Fourteen failures directly harmed 12 patients. Significant predictors of an increased failure rate were: endovascular compared with open repair (incidence rate ratio (IRR) for open repair 0.71, 95 per cent c.i. 0.57 to $0.88 ; P=0.002$ ), thoracic aneurysms compared with other aortic pathologies (IRR 2.07, 1.39 to $3.08 ; P<0.001$ ) and unfamiliarity with equipment (IRR $1.52,1.20$ to $1.91 ; P<0.001$ ). Major failure total was associated with reoperation $(P=0.011)$, major complications $(P=0.029)$ and death $(P=0.027)$

Conclusion: Failure in aortic procedures is frequently caused by issues with team-working and equipment, and is associated with patient harm. Multidisciplinary team training, effective use of technology and new-device accreditation may improve patient outcomes.

\section{+A: Introduction}

It is well known that a significant number of patients come to harm while in hospital ${ }^{1}$. The highest rate of adverse events is in patients undergoing surgical intervention, most notably in those undergoing a major vascular procedure ${ }^{2}$.

Medicine used to be simple, ineffective and relatively safe, whereas it is now complex, effective and potentially dangerous ${ }^{3}$. This is certainly true for major vascular surgery. Patient and technical factors are obvious determinants of outcome, but current thinking emphasizes the role of wider aspects of the surgical system in patient safety ${ }^{1}$. The high-risk nature of vascular procedures in elderly patients with complex co-morbidities, and the importance of technical expertise are well recognized in vascular surgery. Investigation of wider system factors is now warranted, particularly as vascular surgery has undergone significant changes in recent years, with centralization of services in the UK and the rapid development of minimally invasive (endovascular) technologies. These novel strategies for vascular patients have undoubtedly reduced major morbidity and mortality but, to achieve consistently optimal results, continuous emphasis must be placed on optimizing patient 
safety. Single-centre exploratory studies ${ }^{4,5}$ in arterial surgery identified failures related to team communication, surgical equipment and planning, but whether these failures occur at vascular centres throughout the country and, most importantly, their impact on patient outcomes remain unknown. The mechanisms of harm, the determinants of increased failure rates and the impact of system failures on patient outcomes are important information to plan strategies for safety improvement.

The LEAP (Landscape of Error in Aortic Procedures) study is a multicentre, collaborative effort to explore patient safety in vascular surgery. The primary aim of the LEAP study was to define the landscape of surgical system failure, error and inefficiency in open and endovascular aortic surgery. Secondary aims were to investigate determinants of failure, and the relationship between intraoperative failure and patient outcomes.

\section{+A: Methods}

Phase I was a training phase to train teams and establish the reliability of a self-reporting method. In phase II, teams self-reported failures occurring in aortic procedures, the impact of these failures, and patient outcomes. Full research ethics committee (12-LO-0710) and local approvals from the participating organizations were obtained.

\section{+B: Setting and participants}

Participating operating teams were recruited in ten hospitals in England (September 2012 to July 2014). Operating teams consisted of a consultant vascular surgeon, anaesthetists, nurses and support staff, as well as radiology staff (radiologist and/or radiographer) for many procedures; team composition was not the same for all procedures owing to shift patterns and rotation of staff. For the training phase, all adult patients on participating vascular consultants' operating lists were eligible for inclusion in phase 1 . However, in phase 2 , only adult patients undergoing elective or urgent aortic surgery for aneurysm or aorto-occlusive disease were included. Staff and patients provided written, informed consent to participate.

\section{+B: Materials}

Twenty consultant vascular surgeons and their operating teams were trained by a single observer (a senior vascular nurse practitioner) to report intraoperative surgical system failures, errors and inefficiencies using a validated and structured, team-based approach. The Imperial College error 
capture record (ICECAP) is a paper-based tool consisting of several prompts read aloud to structure reporting of failures; its development and validation is described in full elsewhere ${ }^{5}$. Primary failure categories are: equipment, communication, procedure-independent pressures (distractions, team member absence, external pressures), technical, safety awareness and patient-related. Each of the primary categories has a number of secondary fields. For a failure to be recorded on ICECAP, the team had to come to a consensus on whether or not a failure had occurred, and on the category of failure.

\section{+B: Definitions}

A failure was as any event that prevented the procedure from progressing in an ideal manner. This broad definition was a deliberate attempt to capture all relevant safety events. The term failure encompasses different types: failures in the surgical system (system factors), human errors and sources of inefficiency. Failures were to be reported if they occurred between the patient being transferred into the operating theatre and final closure of the wound.

Major and minor failures were defined by their immediate consequences during surgery. Failures that caused intraoperative delay of more than $15 \mathrm{~min}$, caused harm, or placed the patient at significant risk of harm were referred to as major failures. Harm was defined as injury to the patient evidenced by a physiological response to the injury (such as cardiovascular instability), or by the need for further invasive intervention; harm may have occurred during the operation without further sequelae (lasting disability).

\section{+B: Procedure and data collection}

\section{$+C$ : Phase I}

Shift patterns and staff rotation meant that the operating team composition was inconsistent; the trainer therefore undertook multiple visits to each site to capture as many operating staff as possible. The trainer attended a median of 5 (range 4-6) procedures with each consultant vascular surgeon and team, to provide training to all key participating staff. At the beginning of the operating list, the trainer outlined the aims of the study, important definitions and protocol to the operating team. During the procedure, the trainer recorded failures that occurred. At the end of the first procedure with the trainer present, the trainer led the team through the ICECAP debrief and completion of the 
written ICECAP record. Thereafter, a member of the team led the debrief, which took place in the same structured manner on each occasion. The trainer highlighted failures that she recorded to help teams to develop an understanding of the types of failure that should be reported. The trainer encouraged reflection and discussion between team members to enable a consensus to be reached regarding failures to be documented on the ICECAP record.

\section{$+C$ : Phase II}

In the main study phase, each site aimed to collect data independently during 20 consecutive aortic procedures. Sites were asked to complete an aortic procedure log, indicating any interventions that were not recruited, with reasons. For each intervention, patient, procedure and team demographic data were collected, and the primary operator indicated whether he or she was unfamiliar with any of the equipment used. Details of each failure were recorded, including whether or not the patient was harmed, any corrective measures necessary to prevent harm, and the delay caused by the failure. Postoperative complications were recorded for 30 days or until hospital discharge, whichever was sooner.

\section{+B: Handling of data}

Case report forms were collated and information entered into a purpose-built database at the lead site. All failures were reviewed independently by two experienced clinical assessors, and categorized as major or minor, or excluded if they did not meet the study definition. Two independent assessors graded postoperative complications using the Clavien-Dindo system ${ }^{6}$. Grades III, IV and V are referred to as major complications.

\section{$+B$ : Statistical analysis}

Agreement between assessors was assessed by means of Cohen's $\kappa$. Univariable and multivariable Poisson regression analysis was used to compare failure rates (failures per hour) for different patient, procedure and team groups, and the clustering effect resulting from potential variability in reporting styles at the hospital-site level was taken into account by using cluster robust standard errors. The variables included in the regression were: age, sex, ASA fitness grade, aortic pathology, procedure type (open surgical or endovascular), team member experience based on the number of similar operations performed (over 50 versus fewer than 6 similar procedures), and familiarity with 
equipment. The Wilcoxon rank-sum test was used to test for differences in the number of intraoperative failures and patient outcomes. All reported $P$ values are two-sided. $P<0.050$ was deemed to indicate statistical significance. Data were analysed in Stata ${ }^{\circledR}$ version 12 (StataCorp, College Station, Texas, USA) by an independent statistician.

\section{+ A: Results}

\section{+B: Phase I}

Eighty-eight procedures were included in phase I. The failure profiles reported by the trainer and in self-report by operating teams are summarized in Fig. 1. The correlation between the trainer and operating teams for the number of failures identified per procedure was good (Spearman's $\rho=0.766$, $P<0.001)$ and operating teams identified 95 per cent of major failures (18 of 19), indicating that they were able to follow study procedures and assess failure rates reliably.

\section{+B: Phase II}

Following a period of training, 20 consultant vascular surgeons and their operating teams reported failures occurring in 185 elective aortic procedures. Only two urgent patients were recruited and were therefore excluded from analyses; only planned procedures were included. The aortic procedure log was completed poorly and was therefore unhelpful. Teams performing open surgical procedures consisted of the following core members: consultant vascular surgeon, consultant anaesthetist, scrub nurse and support staff. For endovascular interventions, core team members additionally included a consultant radiologist and a radiographer, as is common practice in the UK. All procedures started during daytime hours, although 16.2 per cent (30 of 185) finished after 17.00 hours. Patient, pathology and procedure characteristics are provided in Table 1.

\section{+B: Failure characteristics}

The operating teams recalled 930 failure events in total. Independent assessment excluded 131 events that did not meet the study definition for the following reasons: event did not occur during surgery (43; such as lack of intensive care bed delayed start of procedure); likely anticipated patient-related issue (72; for example, tortuous iliac arteries identified on preoperative imaging); and other (16). Of 799 failures, operating teams identified a median of 3 (i.q.r. 2-6, range $0-23$ ) per procedure. The 
median failure rate was 1.0 (i.q.r. $0.6-2.0$, range $0-6$ per h. There was a significant correlation between the number of failures per procedure and the duration of operation (Spearman's $\rho=0.300, P$ $<0.001)$.

The details of failures are shown in Fig. 2. Overall, most frequent failures related to equipment (33.9 per cent), usually non-availability, ranging from minor issues, such as nonavailability of the desired sheath size, to major failures, for example 'unavailability of the custommade thoracic stent graft, which was believed to be on site but was found not to be available after spinal drain insertion and general anaesthesia'. The primary operator reported unfamiliarity with equipment in 18 procedures (open 1, endovascular 17), of which unfamiliarity with stent-graft devices was reported in 11. Procedure-independent pressures (interruptions and distractions, team member absence and external pressures) were also common (21.7 per cent of all failures).

\section{$+B$ : Consequences of failure}

The consequences of failure were varied. Nearly two-thirds of failures (62.9 per cent) caused intraoperative delays, and 9.8 per cent of failures led to significant delays (more than 15 min during the operation). In total, intraoperative delays accounted for more than $90 \mathrm{~h}$ of unproductive operating time over the 185 procedures. More than one-third of failures (33.8 per cent) necessitated corrective action by the operating teams.

Some 93 (11.6 per cent) of 799 failures were classified as major failures. Most frequent major failures were unanticipated problems related to the patient's anatomy or physiology (33 per cent). Patient-related failures are therefore an important source of unanticipated delay (while the operating team address the problem), and could indicate failures in preoperative planning or preparation. Non-patient-related failures were most commonly communication failures ( 22 per cent of major failures), for example 'radiology staff not informed of start time-delay in starting endovascular phase (45 min) while patient under general anaesthesia'. Of the major communication failures, 65 per cent were due to communication problems between operating sub-teams: between the surgical and radiology teams ( 38 per cent); between the surgical and the anaesthetic teams ( 23 per cent); between other team members including the nursing team (4 per cent). Of the remaining major failures, 17 per cent were technical failures, 13 per cent were equipment-related failures, 7 per cent 
were safety awareness failures (such as checks not done) and 5 per cent were procedure-independent pressures.

Most major failures (83 per cent) led to significant intraoperative delay. Fourteen major failures directly caused harm. Among the 17 per cent of failures that posed significant risk of harm without any associated delays, communication failures were the most common. Of the 14 major failures that led directly to intraoperative harm in 12 patients ( 6.5 per cent of study cohort), communication failures were reported by the team to have led to half of these harm-producing events (Table 2). There were four technical errors that harmed patients directly. Three events related to unanticipated difficulties with the patient's anatomy. Although not a primary aim of the study, review of these failures indicated that additional factors, such as inexperience or a lack of vigilance, may also have played a role; harm was rarely the direct result of a single identifiable cause.

\section{$+B$ : Determinants of failure}

Patient age, sex and ASA grade were not associated with increased failure rates in univariable analyses. There were also no significant differences in failures rates between levels of experience for all professions in the operating team. In multivariable regression, thoracic/arch aneurysms in comparison with other aortic pathologies (incidence rate ratio (IRR) 2.07, 95 per cent c.i. 1.39 to 3.08; $P<0.001)$ were significant predictors of increased failure rates. Of note, two-thirds of patients with thoracic/arch aneurysms in this cohort underwent either carotid subclavian bypass and thoracic endovascular repair (TEVAR) or custom-made TEVAR.

Unfamiliarity with equipment (IRR $1.52,1.20$ to $1.91 ; P<0.001$ ), and endovascular repair compared with open surgical approaches (IRR for open repair $0.71,0.57$ to $0.88 ; P=0.002$ ) were also significant predictors of failure. For major failure, only unfamiliarity with equipment was significantly associated with an increased failure rate (IRR $2.59,1.51$ to $4.28 ; P=0.001$ ).

\section{+B: Association between intraoperative failures and patient outcomes}

Some 152 of 171 procedures were successful technically with no adverse events at $24 \mathrm{~h}$ after surgery (data missing for 14 procedures). Thirteen patients required reoperation, 37 developed major complications, four had a prolonged hospital stay (over 30 days) and seven patients died. The total number of intraoperative failures was significantly higher in procedures requiring an unplanned return 
to theatre: median 5 (i.q.r. $4-10)$ versus $3(2-6)$ for procedures with no further operation $(P=0.037)$. The number of major intraoperative failures was also significantly higher in procedures subsequently requiring reoperation: median $1(0-2.5)$ versus $0(0-1)(P=0.011)$. Significantly greater numbers of major failures were reported during procedures after which the patient developed a major complication: median $0(0-1.5)$ versus $0(0-1)$ for procedures followed by minor or no complications $(P=0.029)$. Similarly, number of major errors was associated with death: median $1(0-3)$ for procedures followed by death versus $0(0-1)$ where the patient survived to discharge $(P=0.027)$ (Fig.

3). There were no differences in the number of intraoperative failures occurring during successful versus unsuccessful procedures (3 (2-6) versus $5(2-6) ; P=0.147)$ or between regular and prolonged (over 30 days) hospital stay (3 (2-6) versus $4(3-5) ; P=0.837)$.

\section{+A: Discussion}

This large study in vascular surgery demonstrates that many avoidable safety failures relate to aspects of the surgical system, in addition to patient factors and technical expertise. This study links intraoperative safety failure with adverse outcomes in patients undergoing aortic surgery. In line with a smaller study of safety failures in cardiac surgery ${ }^{7}$, the present work shows that many failures leading to patient harm stem from failures in the system. Operating teams must recognize potential failures in the surgical system and endeavour to mitigate them.

The National Reporting and Learning System (NRLS) is voluntary system for reporting adverse events and near misses that occur in healthcare. As such, it relies on the details submitted by the reporter and is inherently under-representative of the volume of events that occur ${ }^{8}$. The benefits of NRLS, in the same way as the LEAP study, come from analysing the report to identify themes, which can be targeted for improvement. A retrospective study of NRLS, aiming to identify the causes of patient harm in aortic surgery, demonstrated findings remarkably similar to those of the present study. There is a clear need to address communication and equipment-related failures. A benefit of the ICECAP debriefing approach to identifying intraoperative failures is that it enables learning to take place locally, so that improvement measures can be implemented immediately. 
Intraoperative failures appear to have a significant effect on operating theatre efficiency, as well as patient safety. Nearly two-thirds of errors caused intraoperative delays in procedural workflow, with more than $90 \mathrm{~h}$ lost over 185 procedures. Since the average cost of running an operating theatre exceeds $£ 1200$ per $\mathrm{h}(€ 1443 \text { per h; exchange rate } 28 \text { June } 2016)^{9}$, by avoiding these procedural delays the potential cost savings would be great for each institution.

It has been demonstrated that error rates vary between operation types, with procedures relying on technology, such as those in vascular or cardiac surgery ${ }^{7}$, having the highest rates of total and equipment-related error. The present study has expanded on the findings of previous singlecentre studies, demonstrating that endovascular procedures are consistently associated with more failures than open surgical operations. Although minimally invasive surgery has undoubtedly reduced surgical morbidity and mortality rates, to achieve optimal, safe and efficient outcomes avoidable error must be minimized. The increased rates of error during endovascular versus open surgical procedures may be explained by the rapidly evolving nature of the endovascular field, more challenging technology and the increased number of new devices on the market, resulting in an extensive and changing learning curve. Thoracic/arch aortic aneurysms predicted higher failure rates than infrarenal or juxtarenal aortic procedures, which may be explained by their relative technical difficulty.

In the present study, perceived unfamiliarity with equipment significantly predicted increased rates of intraoperative failures, whereas actual experience (in terms of the number of operations performed) did not. Vascular operating teams are using an increasingly wide range of stent-graft devices in order to treat patients with a broad range of anatomical configurations, and the large number of devices being used is a factor contributing to unfavourable results ${ }^{10}$. Operators planning to introduce a new interventional device have a responsibility to ensure that the introduction of new equipment is accompanied by adequate training, and fulfils safety and financial regulations within their institution. This may include gaining approval from the divisional board and new procedures committee, ensuring that protocols are in place, and ensuring there is a robust structure for audit and evaluation. In reality, however, new devices and equipment are regularly introduced into the operating environment. Currently, it is unclear who is ultimately responsible for training endovascular operators and their teams, whether industry or healthcare institutions, when new devices 
become available for use in patients. New-device training requires attention to improve patient safety.

Communication failures represented a significant proportion of major errors reported in the study, and contributed to half of all intraoperative harm events. Complex aortic procedures demand precise communication and collaboration from increasingly large and multidisciplinary operating teams. This study was undertaken in the UK, where, similar to many European institutions, surgeons and interventional radiologists commonly collaborate to perform endovascular procedures. Dual consultant operating has also become routine practice. Rotation of operating staff is commonplace and, as a result, team members may not work together regularly. Consequently, effective leadership and teamwork are challenging in this high-risk environment, yet training in these essential nontechnical skills is not routine practice, despite the evidence of a relationship between teamwork and patient outcomes ${ }^{11}$.

This study highlights the need to address human factor skills (such as communication, teamworking, leadership) and system factors (equipment planning, provision and maintenance, pressures on the operating team and their environment, provision of training) that may influence patient outcomes in aortic surgery, while continuing to optimize the patient's preoperative condition and technical expertise among surgeons. A substantial proportion of equipment failures were due to nonavailability, failure or configuration, suggesting that there are clear advantages to implementing protocols to reduce equipment-related error. Procedure-specific equipment checks could be incorporated into the WHO's surgical safety checklist ${ }^{12}$, specially adapted for aortic repair.

The provision of teamwork and leadership training for vascular operating teams is crucial to improve patient outcomes. Team simulations have been shown to be a powerful tool for training and improving the technical and non-technical skills required to perform endovascular aortic aneurysm repair and complex cardiovascular procedures, and may be particularly useful for rehearsal of crisis scenarios $^{13}$.

A clear limitation of this study is self-reporting, which is likely to be influenced by cognitive biases, clinical processes (such as production pressure) and individual reporting styles ${ }^{14}$. To minimize the impact of this limitation, the ICECAP tool was used immediately after the procedure to structure 
postoperative team self-reporting of failures, which has been shown to reduce recall bias ${ }^{5}$. Although independent observation is not susceptible to primacy and recency bias effects, and is an alternative to collecting data on intraoperative failures, it is relatively time-consuming and expensive, and may influence operating team behaviours (Hawthorne effect). A further potential limitation of self-report is selective reporting. As the aortic procedure log was completed poorly across participating sites, it is not clear whether consecutive patients were recruited into the study. In an ideal study environment, consistent teams would have been necessary for data reporting. This study aimed to capture real-world intraoperative failures, so the fact that teams were inconsistent provided an honest report. Finally, the number of patients with adverse outcomes was small, so the assessment of between-group differences for failure and patient outcomes was not adjusted for multiple comparisons. The association between intraoperative failures and patient outcomes should be part of future research programmes to further validate these findings.

\section{+ A: Collaborators}

LEAP study collaborators: M. Gohel, P. Hayes (Cambridge University Hospitals NHS Foundation Trust, Cambridge); T. Joseph, A. Sowinski, T. Wilson (North Cumbria University Hospitals NHS Trust, Carlisle Whitehaven); P. Chong, D. Gerrard, A. Croucher (Frimley Health NHS Foundation Trust, Frimley); M. Bown, V. Gokani (National Institute for Health Research (NIHR) Leicester Cardiovascular Biomedical Research Unit and Department of Cardiovascular Sciences, University of Leicester, Leicester); M. McCarthy (University Hospitals of Leicester, Leicester); R. Brightwell, F. Meyer, M. Burrows (Norfolk and Norwich University Hospitals NHS Foundation Trust, Norwich); S. Renton, S. Das, S. Parsapour (North West London Hospitals NHS Trust, London); I. Nordon, S. Baxter, Clifford Shearman (University Hospital Southampton NHS Foundation Trust, Southampton); M. Jenkins (Imperial College Healthcare NHS Trust, London); B. Modarai, S. Clarke (Academic Department of Vascular Surgery, Cardiovascular Division, King's College London; British Heart Foundation Centre of Research Excellence and NIHR Biomedical Research Centre at King's Health Partners, St Thomas' Hospital, London); A. Thompson, S. Cavanagh, A. Gibson, Z. Coleman (York Teaching Hospital NHS Foundation Trust, York).

\section{+A: Acknowledgements}


The authors thank the participants in this study, as well as all attendees at the National Vascular Patient Safety Symposium, and others who helped to shape the outcomes of this report: M. Albayati, L. Allen, P. Avis, L. Bailey, A. Busuttil, B. Byrne, J. Chopade, N. Courtois, A. Davies, L. Desender, A. Forster, M. Hamady, B. Han, T. Hyde, A. James, M. Joseph, R. Kukreja, D. Lewis, M. Li, S. Mason, P. Mayes, M. Metcalfe, M. Mireskandari, A. Morbi, F. Naseem, C. Pettengell, F. Rawf, A. Rolls, N. Rudarakanchana, A. Sharrock, J. Shalhoub, K. Steiner, D. Sykes, C. Thomas, D. Taylor, M. Treherne and I. Yardley. Particular thanks to N. Sevdalis, D. Wilkins and J. Russell.

This work was supported by the Circulation Foundation (President's Early Career Award to C.D.B.); the NIHR (CDRF-2012-03-040 to R.L.); and the NIHR Biomedical Research Centre based at Imperial College Healthcare NHS Trust and Imperial College London. The views expressed in this publication are those of the author(s) and not necessarily those of the funders, the National Health Service, the NIHR or the Department of Health.

\section{$+\mathrm{A}:$ References}

$1 \quad$ Vincent C. Understanding and responding to adverse events. N Engl J Med 2003; 348: $1051-$ 1056.

2 Leape LL, Brennan TA, Laird N, Lawthers A, Localio AR, Barnes BA et al. The nature of adverse events in hospitalized patients. Results of the Harvard Medical Practice Study II. $N$ Engl J Med 1991; 324: 377-384.

3 Chantler C. The role and education of doctors in the delivery of health care. Lancet 1999; 353: $1178-1181$.

4 Albayati MA, Gohel MS, Patel SR, Riga CV, Cheshire NJ, Bicknell CD. Identification of patient safety improvement targets in successful vascular and endovascular procedures: analysis of 251 hours of complex arterial surgery. Eur J Vasc Endovasc Surg 2011; 41: 795802.

5 Mason SL, Kuruvilla S, Riga CV, Gohel MS, Hamady M, Cheshire NJ et al. Design and validation of an error capture tool for quality evaluation in the vascular and endovascular surgical theatre. Eur J Vasc Endovasc Surg 2013; 45: 248-254.

6 Dindo D, Demartines N, Clavien PA. Classification of surgical complications. Ann Surg 2004; 240: $205-213$. 
7 Catchpole KR, Giddings AE, de Leval MR, Godden PJ, Utley M, Gallivan S et al.

Identification of systems failures in successful paediatric cardiac surgery. Ergonomics 2006; 49: $567-588$.

8 Carson-Syevens A, Hibbert P, Avery A, Butlin A, Carter B, Cooper A et al. A cross-sectional mixed methods study protocol to generate learning from patient safety incidents reported from general practice. BMJ Open 2015; 5: e009079.

$9<$ EPATH $>$ NHS Institute for Innovation and Improvement. The Productive Operating Theatre. Improving Quality and Efficiency in the Operating Theatre. A Lifeline for Financial Leaders.

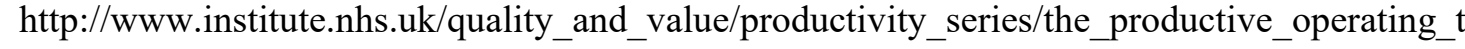
heatre.html [accessed 8 May 2016].

10 Cochennec F, Kobeiter H, Gohel M, Majewski M, Marzelle J, Desgranges P et al. Impact of intraoperative adverse events during branched and fenestrated aortic stent grafting on postoperative outcome. J Vasc Surg 2014; 60: 571-578.

11 Neily J, Mills PB, Young-Xu Y, Carney BT, West P, Berger DH et al. Association between implementation of a medical team training program and surgical mortality. JAMA 2010; 304: $1693-1700$.

12 Haynes AB, Weiser TG, Berry WR, Lipsitz SR, Breizat A-HS, Dellinger EP et al. Safe Surgery Saves Lives Study Group. A surgical safety checklist to reduce morbidity and mortality in a global population. N Engl J Med 2009; 360: 491-499.

13 Arriaga AF, Bader AM, Wong JM, Lipsitz SR, Berry WR, Ziewacz JE et al. Simulation-based trial of surgical-crisis checklists. N Engl J Med 2013; 368: 246-253.

14 Oken A, Rasmussen MD, Slagle JM, Jain S, Kuykendall T, Ordonez N et al. A facilitated survey instrument captures significantly more anesthesia events than does traditional voluntary event reporting. Anesthesiology 2007; 107: 909-922. 
Fig. 1 Profile of failures identified through prospective observation by the trainer and structured team self-reporting during the training phase

Fig. 2 Causes of failure in 185 aortic procedures by primary category and subcategory Fig. 3 Association between major failures per procedure and patient outcomes: a unplanned return to the operating theatre, $\mathbf{b}$ postoperative complications and $\mathbf{c}$ in-hospital mortality. Median values (bold line), i.q.r. (box) and $1.5 \times$ i.q.r. (whiskers) are shown; outliers are represented by symbols. $* P=$ $0.011, \dagger P=0.029, \ddagger P=0.027$ (Wilcoxon rank-sum test, not adjusted for multiple comparisons) 
Table 1 Patient and procedure data in phase II

\begin{tabular}{|c|c|}
\hline & $\begin{array}{c}\text { No. of patients } \\
\quad(n=185)\end{array}$ \\
\hline \multicolumn{2}{|l|}{ Patient demographics } \\
\hline Mean age (years)* & $73(10)(37-100)$ \\
\hline Male sex & 153 of $180(85.0)$ \\
\hline \multicolumn{2}{|l|}{ ASA fitness grade } \\
\hline I & 2 of $179(1.1)$ \\
\hline II & 44 of $179(24.6)$ \\
\hline III & 119 of $179(66.5)$ \\
\hline IV & 14 of $179(7.8)$ \\
\hline \multicolumn{2}{|l|}{ Primary aortic pathology } \\
\hline Infrarenal abdominal AAA & 111 of $182(61.0)$ \\
\hline Juxtarenal abdominal AAA & 30 of $182(16.5)$ \\
\hline Thoracic/arch aortic aneurysm & 8 of $182(4.4)$ \\
\hline Thoracoabdominal aortic aneurysm & 15 of $182(8.2)$ \\
\hline Occlusive disease & 9 of $182(4.9)$ \\
\hline Other & 9 of $182(4.9)$ \\
\hline \multicolumn{2}{|l|}{ Procedures } \\
\hline Open infrarenal AAA repair & $21(11.4)$ \\
\hline Open juxtarenal aortic aneurysm repair/type IV aortic aneurysm repair & $9(4.9)$ \\
\hline Open aortoiliac bypass & $7(3.8)$ \\
\hline Open aortofemoral bypass & $7(3.8)$ \\
\hline EVAR - conventional & $69(37.3)$ \\
\hline EVAR - additional complexity $\dagger$ & $18(9.7)$ \\
\hline Branched EVAR/fenestrated EVAR - conventional & $23(12.4)$ \\
\hline Fenestrated EVAR - additional complexity $\dagger$ & $4(2.2)$ \\
\hline Thoracic EVAR - conventional & $8(4.3)$ \\
\hline Thoracic EVAR - additional complexity $\dagger$ & $7(3.8)$ \\
\hline Visceral hybrid repair & $2(1.1)$ \\
\hline Other & $10(5.4)$ \\
\hline
\end{tabular}

Values in parentheses are percentages unless indicated otherwise; *values are mean(s.d.) (range).

$\dagger$ Endovascular procedure with additional intervention besides femoral cut down for arterial access. AAA, abdominal aortic aneurysm; EVAR, endovascular aneurysm repair. 
Table 2 Illustrative quotes from Imperial College error capture record records for major failure

'Misleading communication led to wrong clamps being taken off the graft...substantial blood loss, which was 'hidden' and went unnoticed leading to severe hypotension in a patient with significant co-morbidity' (hybrid open visceral artery retrograde revascularizsation and endovascular thoracoabdominal aneurysm repair)

'Communication failure and discord between surgeons and scrub nurse during bleeding. Scrub nurse unfamiliar with use of pledgeted sutures to repair internal iliac vein injury.

Miscommunication led to a further tear in vein as sutures were pulled out. Patient became acutely unstable' (EVAR with surgical ligation of IIA)

'Wrong sized limb placed necessitating embolization of IIA' (EVAR and left to right femorofemoral artery crossover graft)

'Wrong incision due to lack of communication between radiology and surgical teams' (aortouniiliac and femorofemoral artery crossover graft)

'Lack of communication between surgeons and anaesthetist regarding degree of blood loss.

Change of consultant anaesthetist mid-procedure - patient not transfused after initial 2 units.

Miscommunication with blood transfusion laboratory - delay in receiving cross-matched blood for hypovolaemic patient' (thoracic EVAR)

EVAR, endovascular aneurysm repair; IIA, internal iliac artery. 